\title{
Nuclear Science User Facility: Overview of Neutron Irradiation Activities
}

\author{
Brenden Heidrich
}

November 2017

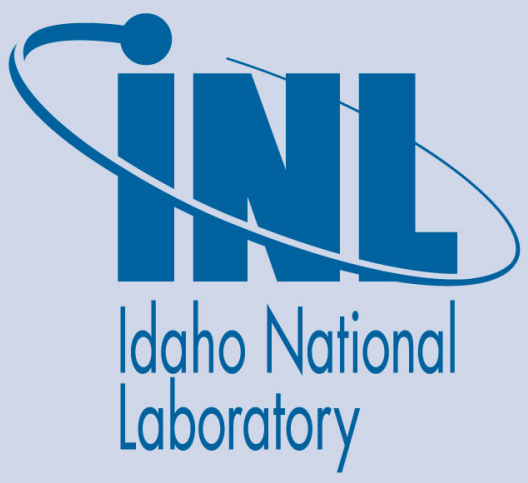

The INL is a U.S. Department of Energy National Laboratory operated by Battelle Energy Alliance 


\title{
Nuclear Science User Facility: Overview of Neutron Irradiation Activities
}

\author{
Brenden Heidrich \\ November 2017 \\ Idaho National Laboratory \\ Idaho Falls, Idaho 83415 \\ http://www.inl.gov \\ Prepared for the \\ U.S. Department of Energy \\ Under DOE Idaho Operations Office \\ Contract DE-AC07-05ID14517
}




\section{Nuclear Science User Facility}

\section{Overview of Neutron Irradiation Activities}

Brenden Heidrich, Ph.D. Chief Irradiation Scientist, NSUF

Annual NSUF Program Review DOE Headquarters Germantown, MD November 13, 2017 


\section{Outline}

1. NSUF Neutron Irradiation Capabilities

- NSUF Neutron Irradiation Capability Demand

2. NSUF Neutron Irradiation Experiments

- Challenges: specimen readiness

3. Neutron Irradiation Toolkit Development

- Path forward 
Nuclear Science User Facilities

\section{NEUTRON IRRADIATION CAPABILITIES}




\section{NEAC Neutron Irradiation Capabilities Conclusions}

\section{Conclusions:}

1. U.S. Rx have sufficient thermal flux for fuels irradiations.

2. U.S. Rx have insufficient fast flux for accelerated testing of advanced reactor materials.

3. U.S. Rx are not currently capable of irradiating fuels and materials in thermal, hydraulic, mechanical, and chemical environments representative of advanced liquid-metal or molten-salt reactors.

4. U.S. Rx are $\geq 50$ years old.

Recommendations:

1. Utilize the Gen IV Intl Forum DB to update the NSUF NEID.

2. Engage in communication with intl. facilities (incl. Russia and India).

3. Proceed immediately with pre-conceptual design planning activities to support a new test reactor. 


\section{Global Research \& Test Reactors}

\begin{tabular}{|c|c|c|c|c|}
\hline Reactor & Country & $\begin{array}{c}\text { Thermal Flux } \\
\qquad\left[10^{14} \mathrm{nv}\right]\end{array}$ & $\begin{array}{l}\text { Fast Flux } \\
{\left[10^{14} \mathrm{nv}\right]}\end{array}$ & Utilization \\
\hline JOYO & Japan & & 40 & Material, fuel \\
\hline BOR-60 & Russia & 2 & 35 & Material, isotopes \\
\hline HFIR & US-TN & 25 & 10 & Isotopes, beam, fuel, material \\
\hline BR-2 & Belgium & 10 & 7.1 & Fuel \& material, isotopes \\
\hline ATR & US-ID & 10 & 5 & Material, fuel, isotopes \\
\hline HANARO & S. Korea & 4.5 & 3 & Isotope, beam, fuel, material \\
\hline SAFARI-1 & S. Africa & 2.4 & 2.8 & Isotopes, beam, radiography \\
\hline NBSR & US-MD & 4 & 2 & Neutron scattering, beam \\
\hline MITR & US-MA & 0.5 & 1.7 & Material, beam, silicon \\
\hline MURR & US-MO & 6 & 1 & Material, silicon, isotopes \\
\hline HBWR & Norway & 1.5 & 0.8 & Material, fuel, loops \\
\hline PULSTAR & US-NC & 0.20 & 0.02 & Isotope, silicon, beams \\
\hline OSURR & US-OH & 0.12 & 0.05 & Sensors, high-temp testing \\
\hline ACRR & US-NM & & & Transient testing, rad effects \\
\hline SPR-CX & US-NM & & & Critical facility \\
\hline
\end{tabular}




\section{Advanced Test Reactor}

\section{$\underline{\text { High Flux Isotope Reactor }}$}

Small B Position

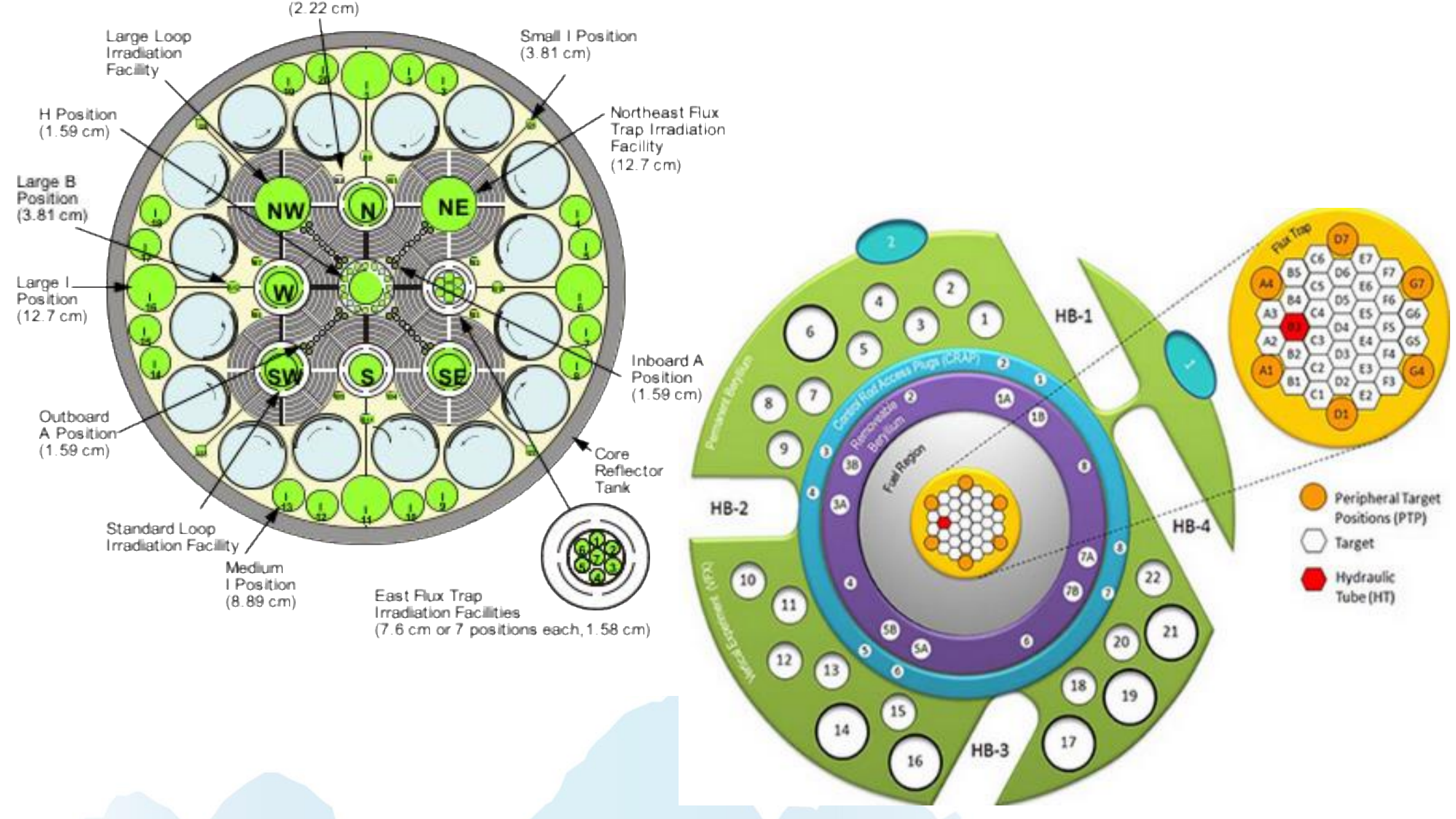




\section{Belgian Reactor-2}

- Multiple experiment vehicles

- Static Capsules \& Instrumented Leads

- Pressurized water capsules for fuel tests (PWC)

- PWR loop (CALLISTO)

- Sodium loop (IPSL)

- SS and transient tests

- SS: $600 \mathrm{w} / \mathrm{cm}^{2}$

- TT: $100 \mathrm{~W} / \mathrm{cm} / \mathrm{min}$

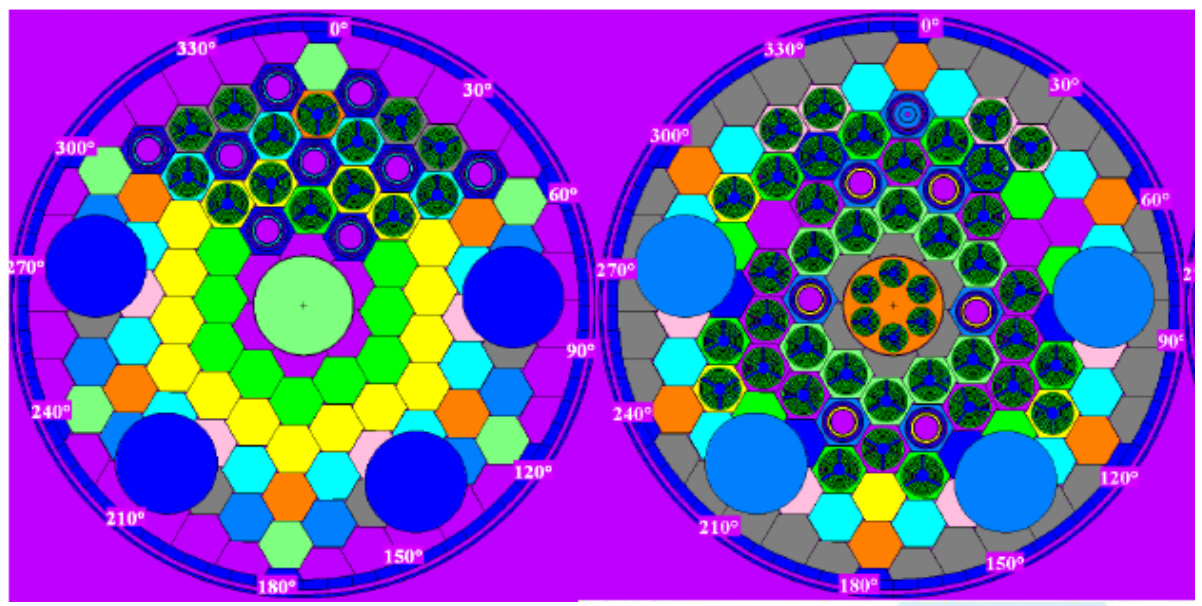

\section{MIT Reactor-II}

- Inert gas (He/Ne mix) irradiation:

- Instrumented (ICSA facility)

- High temperature $\left(>900^{\circ} \mathrm{C}\right)$

- Forced-circulation coolant loops for LWR conditions,

- Custom facilities for unique conditions (molten F salts).

- Thermal flux $0.4 \times 10^{14} \mathrm{n} / \mathrm{cm}^{2}-\mathrm{s}$

- Fast flux $1.2 \times 10^{14} \mathrm{n} / \mathrm{cm}^{2}-\mathrm{s}$.

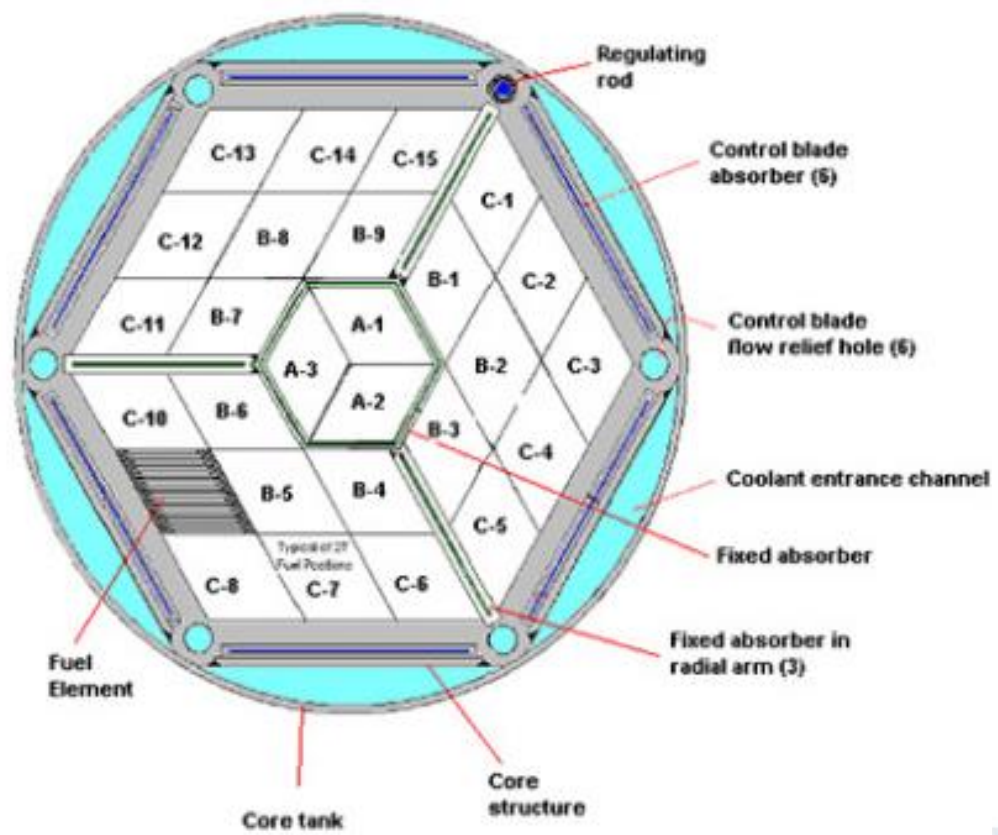




\section{NCSU PULSTAR Reactor}

- $1 \mathrm{MW}_{\text {th }}$ (upgrading to $2 \mathrm{MW}$ )

- $4 \%$ enriched pellets with Zirc-2 clad

- Sample sizes range: 3.175-8.89 cm

- Thermal Flux range: $10^{12}-10^{13} \mathrm{n} / \mathrm{cm}^{2} / \mathrm{s}$

- Fast Flux range: $5 \times 10^{9}-10^{12} \mathrm{n} / \mathrm{cm}^{2} / \mathrm{s}$

- Capabilities

- Positron intense beam facility

- Neutron powder diffraction facility

- Neutron imaging facility

- Ultra-cold neutron source

- TRISO fission gas sampling

\section{Ohio State University Research Reactor}

500kW LEU pool-type URR

- CIF, AIF \& Rabbit system in-core

- 7 \& 10" Dry Tubes at core edge

- Temperature control from 4-1873K

\begin{tabular}{|c|c|c|}
\hline Facility & $\begin{array}{c}\text { Thermal Flux } \\
{[\mathrm{nv}<0.5 \mathrm{eV}]}\end{array}$ & $\begin{array}{c}\text { Fast Flux } \\
{[\mathrm{nv}>1 \mathrm{Mev}]}\end{array}$ \\
\hline CIF & $1.4 \mathrm{E}+13$ & $4.7 \mathrm{E}+12$ \\
\hline $10 "$ Dry Tube & $3.1 \mathrm{E}+11$ & $1.6 \mathrm{E}+11$ \\
\hline
\end{tabular}
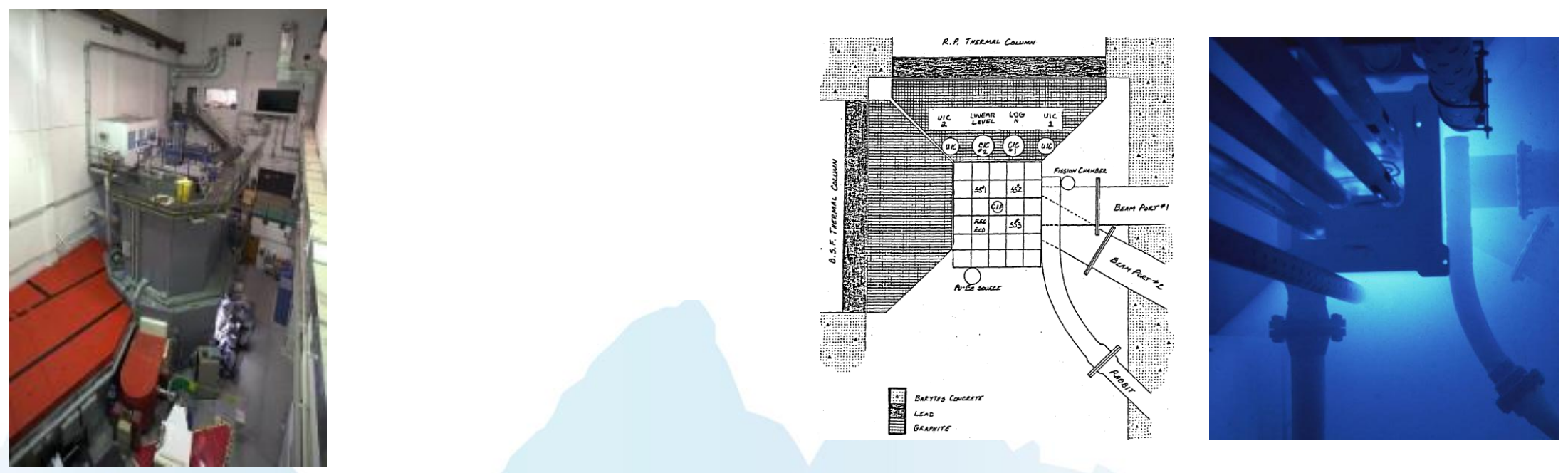


\section{$\underline{\text { Transient Reactor Test (INL) }}$}

- Induce intense fission heating in the nuclear fuel being tested.

- Test nuclear reactor fuels under severe reactor-accident conditions.

- Provide nondestructive test data through neutron radiography of fuel samples.

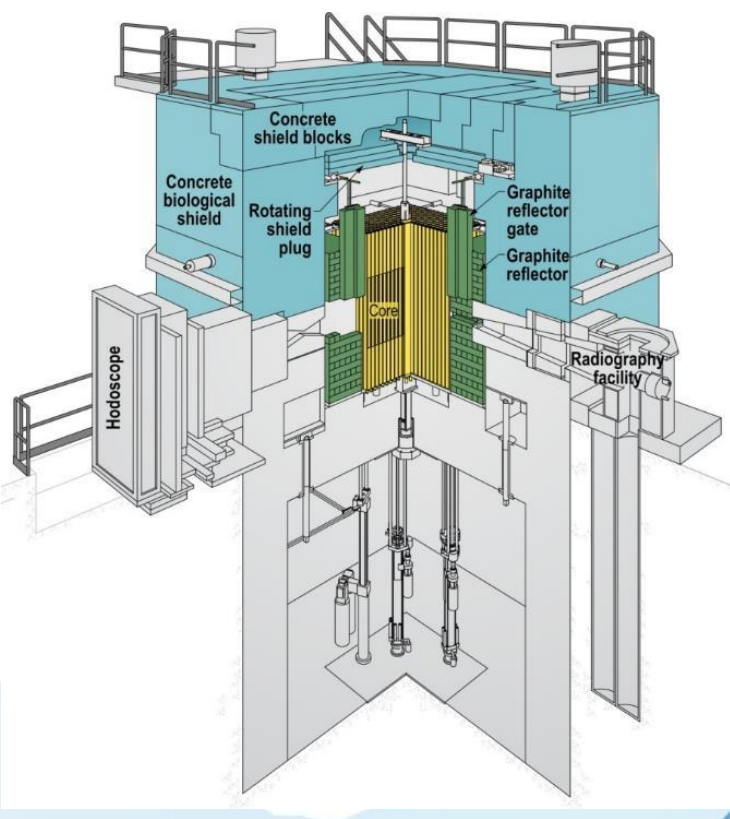

\section{Annular Core Research Reactor}

- High-flux pulsing reactor w/ tunable spectrum

- 9" diameter central cavity

- 20" diameter FREC-II cavity

- $10^{15}$ nvt E>1 MeV pulse

- $6.4 \times 10^{15} \mathrm{nvt}$ total MeV pulse

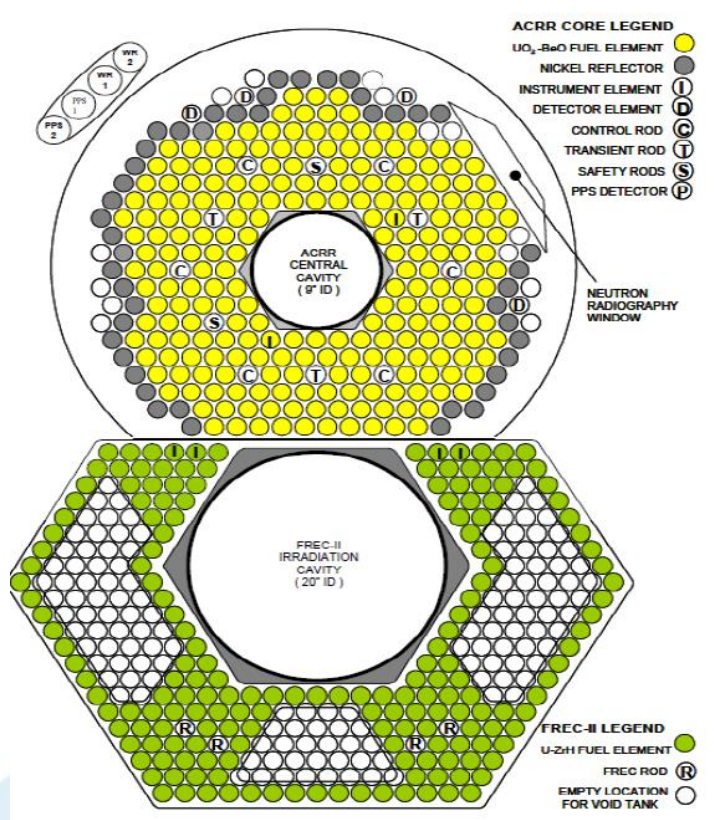




\section{ATR Critical Facility}

- $0.1 \mathrm{~kW}_{\text {th }}$ (typical) $5 \mathrm{~kW}_{\text {th }}$ (max)

- Thermal Flux $2.3 \times 10^{10} \mathrm{n} / \mathrm{cm}^{2} / \mathrm{s}$

- Fast Flux $\quad 0.7 \times 10^{10} \mathrm{n} / \mathrm{cm}^{2} / \mathrm{s}$

- ATR-C provides physics data for:

- worth and calibration of control elements,

- excess reactivity and charge lifetimes,

- thermal and fast neutron distributions,

- gamma heat generation rates

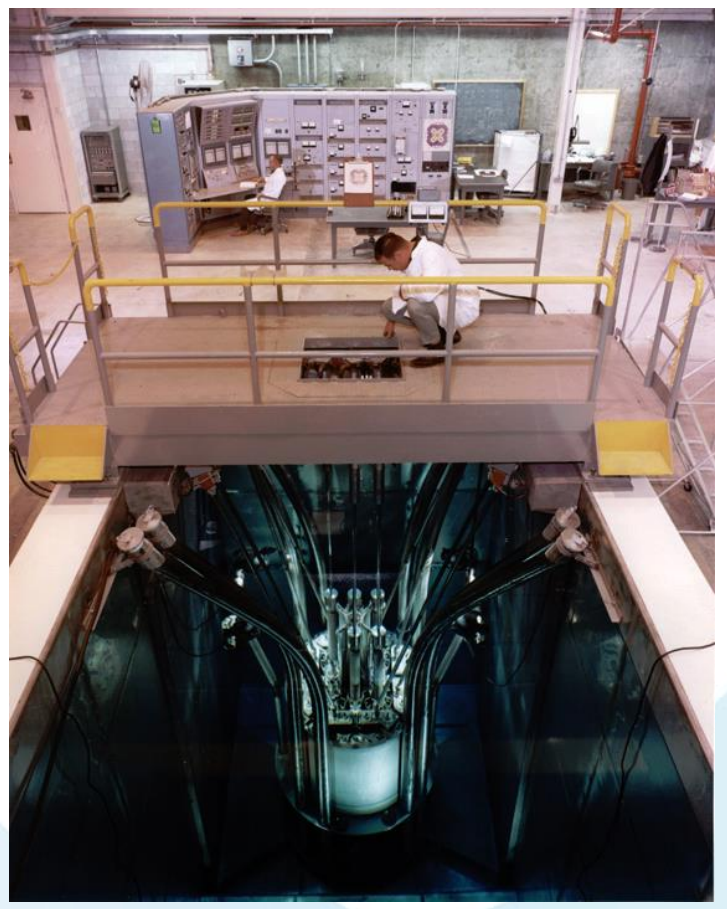

\section{SPR-CX Critical Facility}

- $7.2 \%$ LEU UO 2

- BUCCX (BurnUp Credit Critical Experiment)

- 7uPCX (Seven Percent Critical

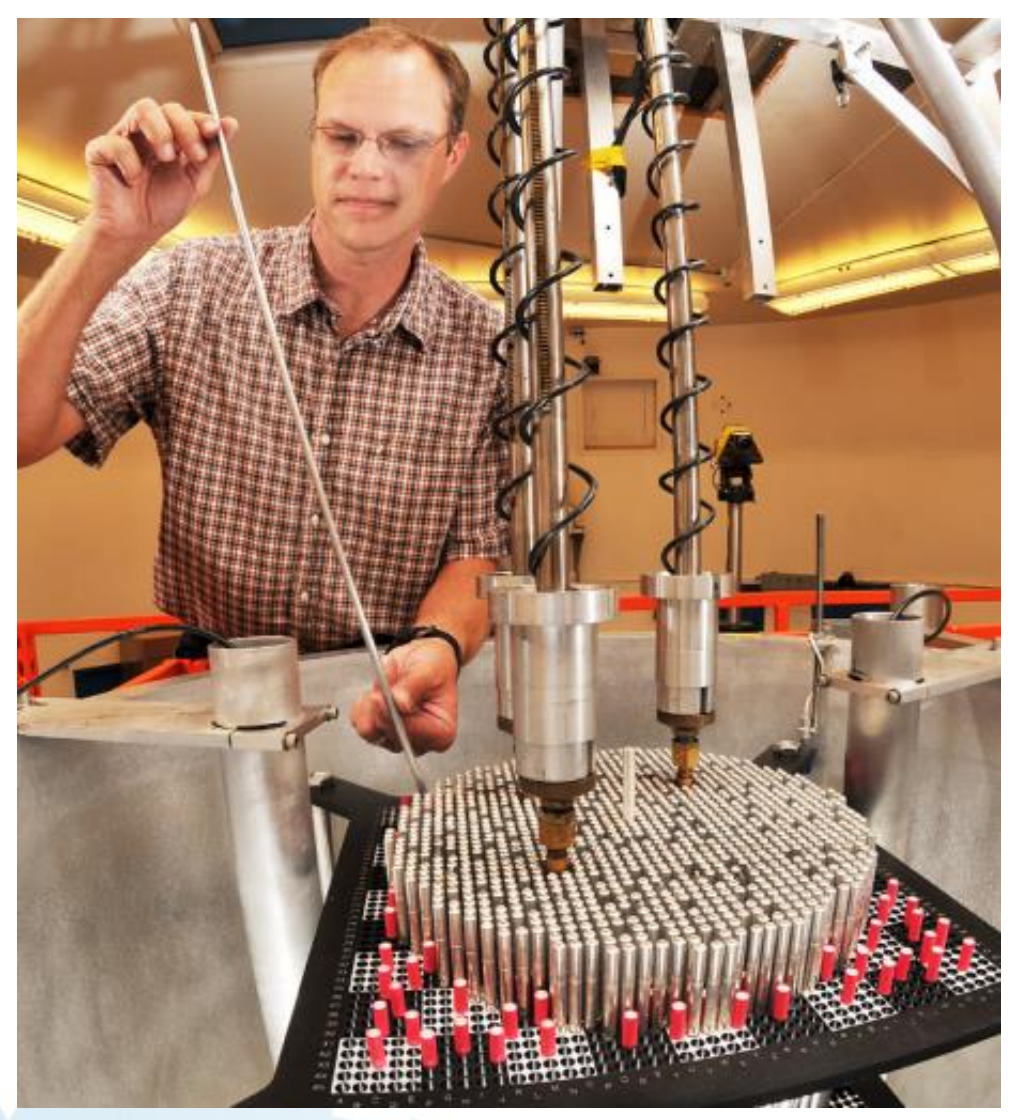




\section{Nuclear Radiography Reactor}

- 250kW TRIGA Reactor

- Purpose: Non-destructively interrogate internals

- Application:

- Evaluate fuel integrity and movement

- Hydriding in LWR cladding

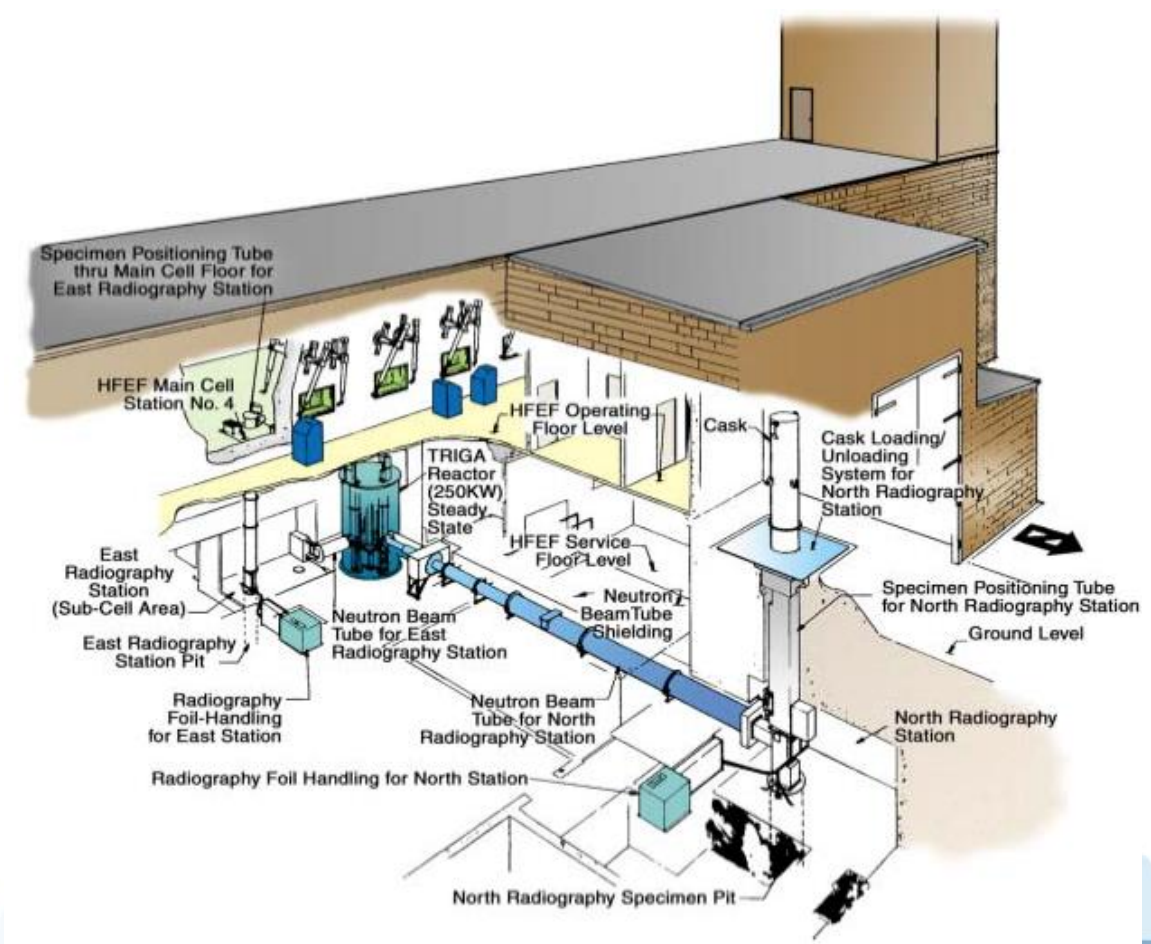

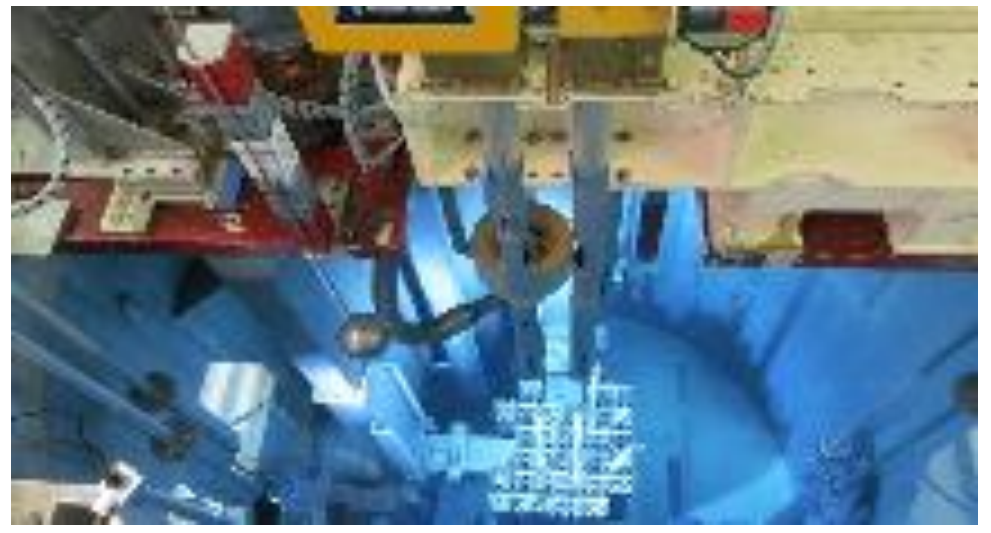

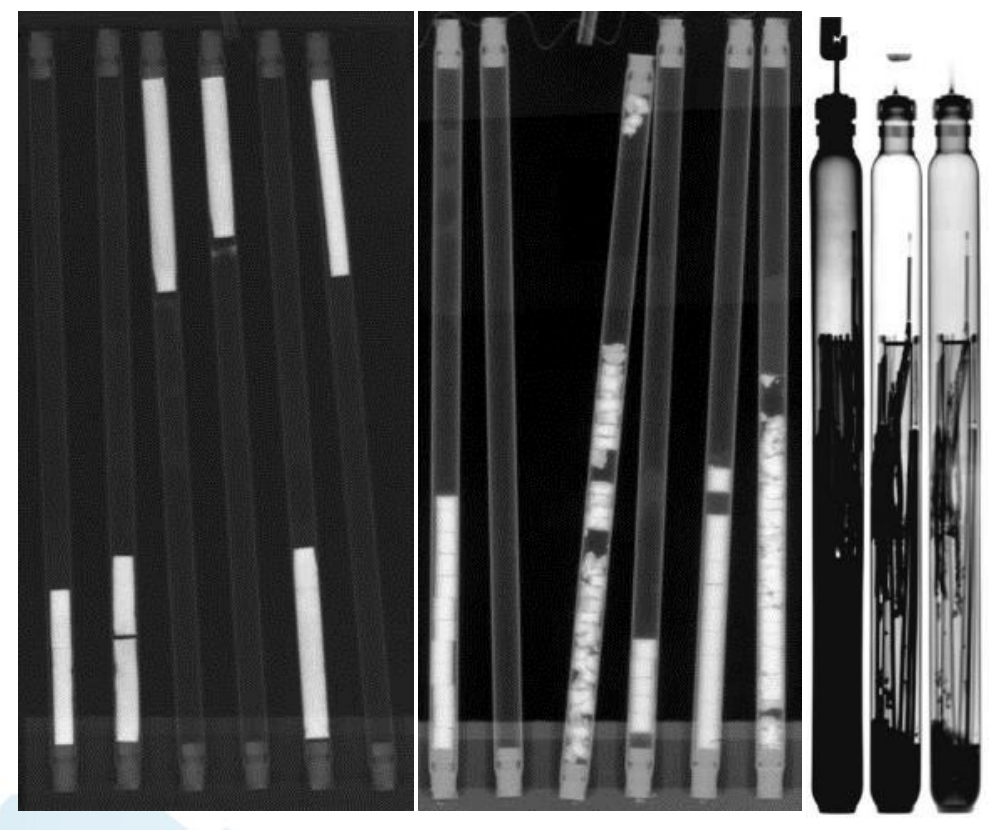




\section{What are the remaining gaps?}

- High(er) fast flux

- LWR loop (more)

- Non-LWR loop

- Pulsing (TRIGA) 


\section{National Bureau of Standards Reactor}

- HEU Fuel: $93 \%{ }^{235} \mathrm{U}_{3} \mathrm{O}_{8}+\mathrm{Al}$ with $\mathrm{D}_{2} \mathrm{O}$ Coolant, Moderator, Reflector

- Fuel cycle: 38 days with a peak flux of $3.5 \times 10^{14} \mathrm{n} / \mathrm{cm}^{2} / \mathrm{sec}$

- 9 radial thermal neutron beams, 5 "rabbits" and 10

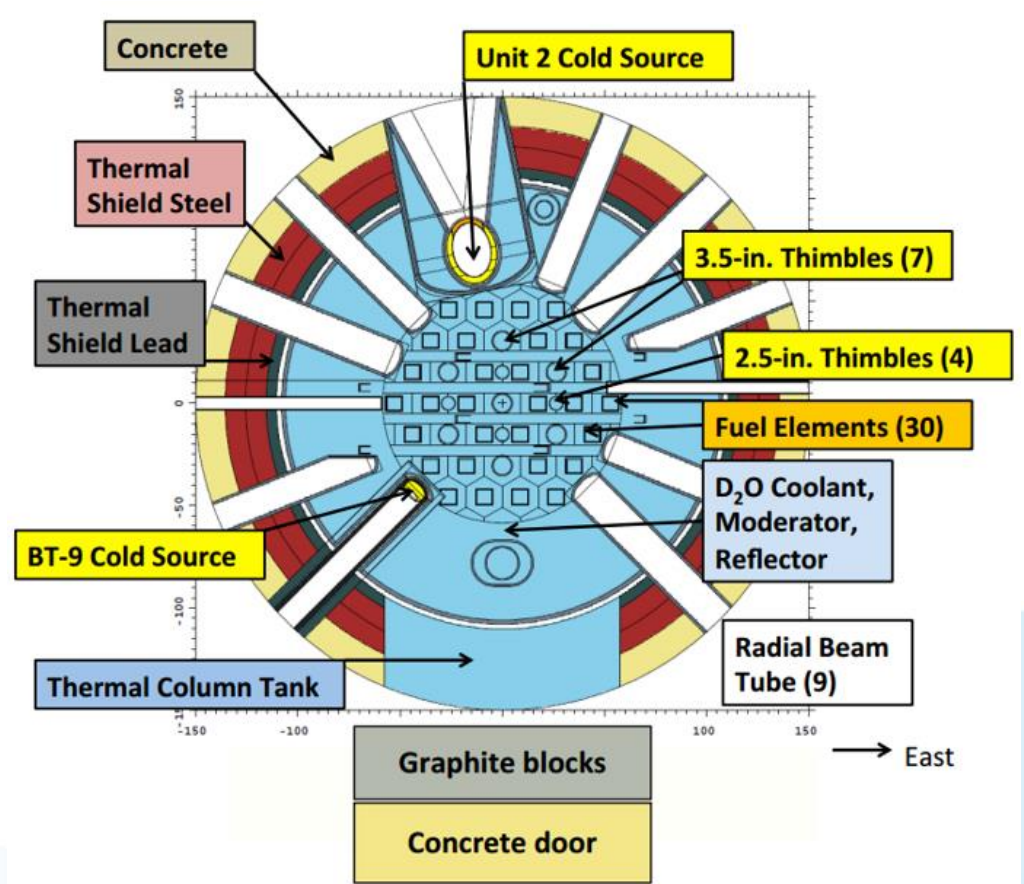

\section{University of Missouri- Columbia Reactor}

- 8 93\% HEU fuel elements

- $10 \mathrm{MW}, 53 \mathrm{C}, 85$ psia

- 3 flux trap locations, 15 reflector locations

- 6 beam ports for neutron scattering

- Primary mission is isotope production

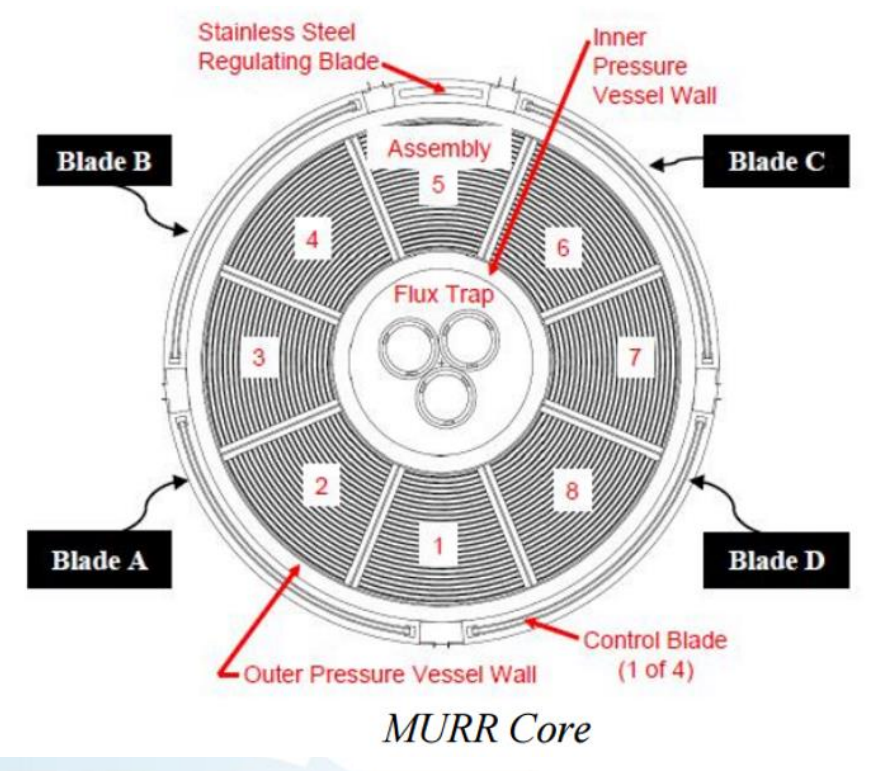


Nuclear Science User Facilities

\section{FY2018 CINR IRRADIATION PROPOSALS}




\section{NSUF Irradiation Utilization (by CINR)}

\begin{tabular}{|l|c|c|c|}
\hline Reactor & FY15 FOA & FY16 FOA & FY17 FOA \\
\hline ATR (INL) & 1 & $3+$ SAM1 & $1+$ SAM2(?) \\
\hline HFIR (ORNL) & 0 & 2 & 2 \\
\hline MITR-II (MIT) & 1 & 1 & 2 \\
\hline PULSTAR (NCSU) & 0 & 1 & 2 \\
\hline BR-2 (SCK-CEN) & 0 & 3 & 0 \\
\hline
\end{tabular}

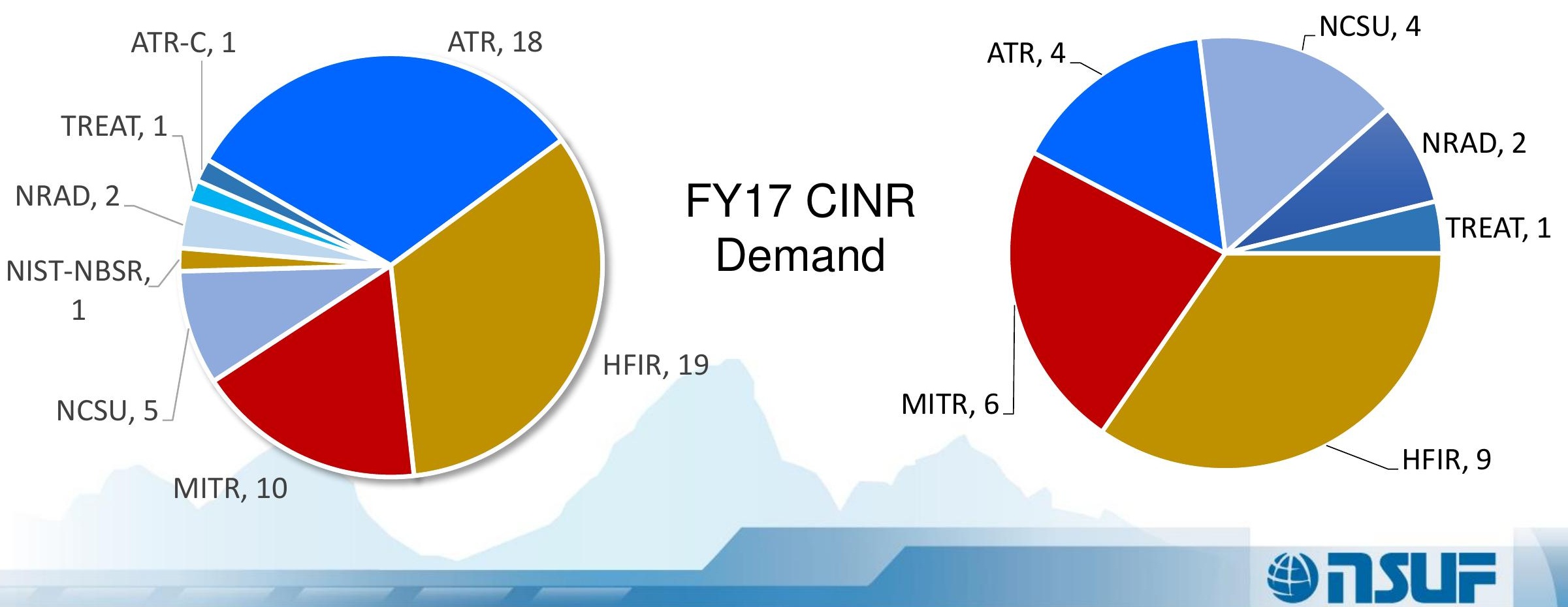




\section{Neutron Irradiation Proposals (FY 2018 access requests)}

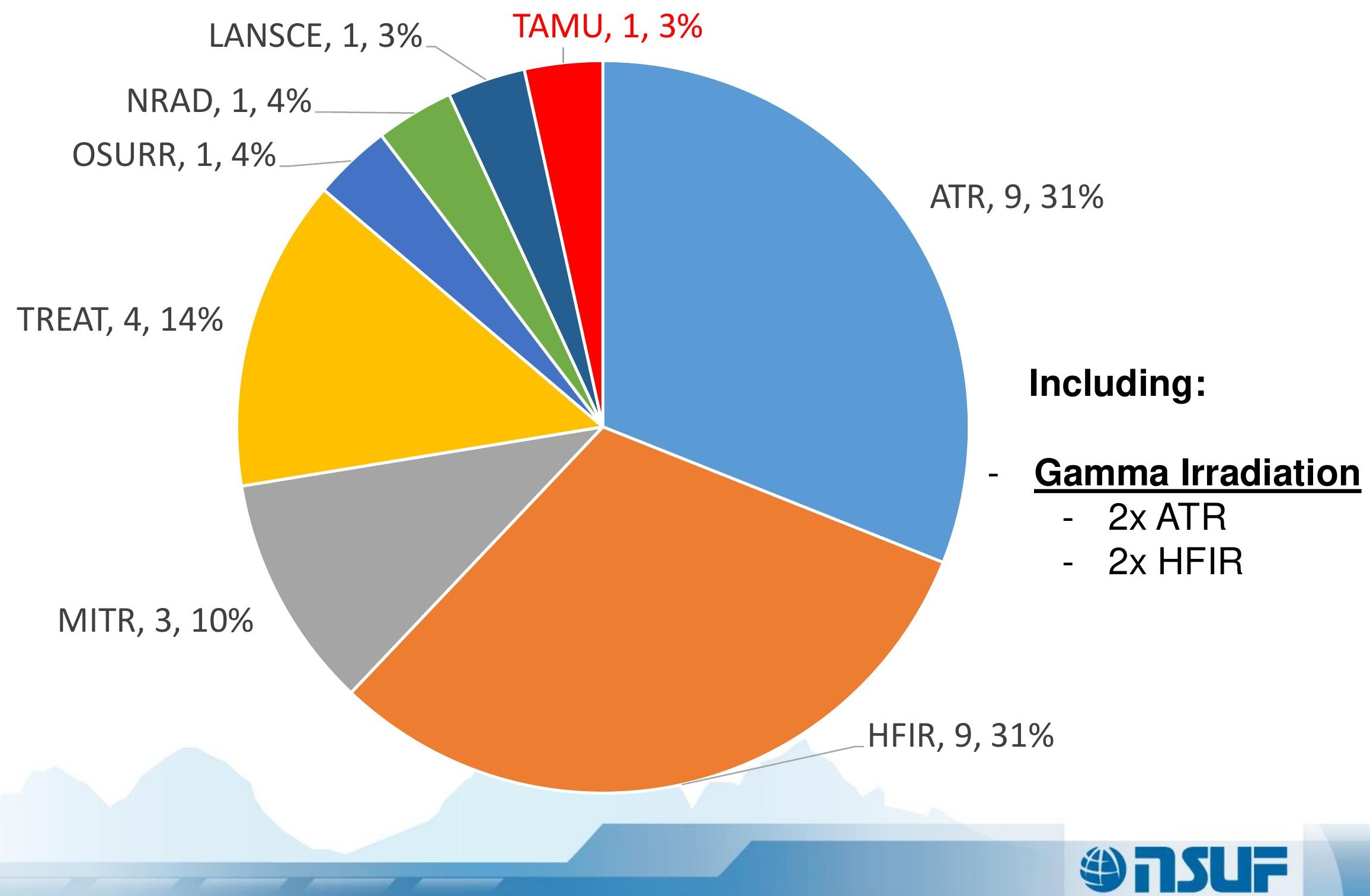




\section{Irradiation Requests by Workscope}

- Likely to fund only:

- one in each R\&D workscope and

- One or two in the NSUF2.1 \& 2.2 workscopes.

\begin{tabular}{|r|c|c|c|c|c|}
\hline & 1.1 & 1.2 & NEAMS 2 & 2.1 & 2.2 \\
\hline ATR & & 5 & 2 & 2 & \\
\hline HFIR & 4 & 3 & & 2 & \\
\hline MITR & 2 & 1 & & & \\
\hline TREAT & 1 & & 2 & & 1 \\
\hline OSURR & 1 & & & & \\
\hline NRAD & & & & 1 & \\
\hline LANSCE & & & & 1 & \\
\hline TAMU & 1 & & & & \\
\hline Total & 9 & 9 & 4 & 6 & 1 \\
\hline
\end{tabular}




\section{ATR Position Strategy}

- Specific call in CINR

-3D programming of ATR

- Fill in the gap with SAMs 
Nuclear Science User Facilities
NEUTRON IRRADIATION PROJECTS 


\section{Nuclear Fuels}

\begin{tabular}{|c|c|c|c|c|c|c|}
\hline Number & Institution & PI & Purpose & Materials & Reactor & Status \\
\hline $10-242-2$ & $\begin{array}{l}\text { Univ. Central } \\
\text { Florida }\end{array}$ & $\begin{array}{l}\text { Yongho } \\
\text { Sohn }\end{array}$ & $\begin{array}{l}\text { Metallic fuels at low } \\
\text { fluences (AFCl, } \mathrm{M}^{3} \text { ) }\end{array}$ & $\begin{array}{l}\text { Diffusion } \\
\text { Couples }\end{array}$ & ATR (HSIS) & On hold \\
\hline $10-242-3$ & $\begin{array}{l}\text { Univ. Central } \\
\text { Florida }\end{array}$ & $\begin{array}{l}\text { Yongho } \\
\text { Sohn }\end{array}$ & $\begin{array}{l}\text { Metallic fuels at low } \\
\text { fluences (AFCl, } \mathrm{M}^{3} \text { ) }\end{array}$ & DC, TEM, MPC & ATR (B8) & $\begin{array}{l}\text { Running, done } \\
\text { in } 2 Q 18\end{array}$ \\
\hline $10-269-1$ & $\begin{array}{l}\text { Boise State } \\
\text { Univ. (Utah } \\
\text { State Univ.) }\end{array}$ & Darryl Butt & $\mathrm{U}_{3} \mathrm{Si}_{2}$ fuel interaction & $\begin{array}{l}\text { Diffusion } \\
\text { couples }\left(\mathrm{U}_{3} \mathrm{Si}_{2}\right. \\
\mathrm{Zr}, \mathrm{FeCrAl}, \mathrm{SiC})\end{array}$ & ATR (I14) & $\begin{array}{l}\text { Running, done } \\
\text { post-CIC }\end{array}$ \\
\hline 17-12985 & $\begin{array}{l}\text { Electric } \\
\text { Power } \\
\text { Research } \\
\text { Institute }\end{array}$ & $\begin{array}{l}\text { Ken Yueh } \\
\text { (EPRI) \& } \\
\text { Michelle } \\
\text { Bales (NRC) }\end{array}$ & $\begin{array}{l}\text { Irradiation, and PIE } \\
\text { of Ultra High Burnup } \\
\text { Fuel (LOCA perf.) }\end{array}$ & $\begin{array}{l}\text { Irradiated } \mathrm{UO}_{2} \\
\text { from } \mathrm{LWR}\end{array}$ & ATR (CFT) & $\begin{array}{l}\text { Specimen } \\
\text { selection 1Q18 }\end{array}$ \\
\hline $\begin{array}{l}\text { BR2-2 } \\
\text { DISECT }\end{array}$ & $\begin{array}{l}\text { Purdue and } \\
\text { INL }\end{array}$ & $\begin{array}{l}\text { Dan Wachs } \\
\text { and Maria } \\
\text { Okuniewski }\end{array}$ & $\begin{array}{l}\text { Separate effects } \\
\text { testing under } \\
\text { controlled } \\
\text { temperatures }\end{array}$ & $\begin{array}{l}\text { Foils and } \\
\text { matchsticks of } \\
\text { U-Zr and U-Mo } \\
\text { fuel }\end{array}$ & BR2 & $\begin{array}{l}\text { Mature } \\
\text { design, } \\
\text { fabrication } \\
\text { underway }\end{array}$ \\
\hline $\begin{array}{l}\text { BR2-3 } \\
\text { ATTICUS }\end{array}$ & BSU \& INL & $\begin{array}{l}\text { Darryl Butt } \\
\text { \& Jason } \\
\text { Harp }\end{array}$ & $\begin{array}{l}\mathrm{U}_{3} \mathrm{Si}_{2} \text { fuel water } \\
\text { interaction and } \\
\text { corrosion }\end{array}$ & $\begin{array}{l}\text { Fuel pins of } \\
\mathrm{U}_{3} \mathrm{Si}_{2} \& \\
\mathrm{UN} / \mathrm{U}_{3} \mathrm{Si}_{2}\end{array}$ & BR2 (PWC) & $\begin{array}{l}\text { INL FDR due } \\
3 Q 18\end{array}$ \\
\hline
\end{tabular}




\section{Advanced Manufactured Materials}

\begin{tabular}{|c|c|c|c|c|c|c|}
\hline Number & Institution & PI & Purpose & Materials & $\mathbf{R x}$ & Status \\
\hline $\begin{array}{l}\text { EPRI-ZG } \\
(2010)\end{array}$ & $\begin{array}{l}\text { Electric } \\
\text { Power } \\
\text { Research } \\
\text { Institute }\end{array}$ & & $\begin{array}{l}\text { Radiation- } \\
\text { induced growth } \\
\text { of LWR cladding }\end{array}$ & $\begin{array}{l}\text { Various pre-hydrided } \\
\text { zirconium alloys for } \\
\text { LWR cladding }\end{array}$ & $\begin{array}{l}\text { ATR } \\
\text { (A13- } \\
16)\end{array}$ & $\begin{array}{l}\text { - TEM analysis } \\
\text { completed } \\
20 \& 30 \mathrm{dpa} \\
\text { in ATR }\end{array}$ \\
\hline $15-8242$ & $\begin{array}{l}\text { Boise State } \\
\text { Univ. } \\
\text { (Purdue) }\end{array}$ & $\begin{array}{l}\text { Janelle } \\
\text { Wharry }\end{array}$ & $\begin{array}{l}\text { HIP-PIM metals } \\
\text { vs. cast/forged } \\
\text { (weldability \& } \\
\text { inspectability) }\end{array}$ & $\begin{array}{l}\text { TEM \& tensile for } \\
\text { 625, 690, Grade 91, } \\
\text { 304L, 316L SA } 508\end{array}$ & $\begin{array}{l}\text { ATR } \\
\text { (A4-6) }\end{array}$ & $\begin{array}{ll}\text { - } & \text { FDR comp. } \\
\text { - } & \text { Insert 2Q18 } \\
\text { - } & \text { Done 3Q19 }\end{array}$ \\
\hline $\begin{array}{l}15-10537 \\
\text { N-SERT }\end{array}$ & $\begin{array}{l}\text { Idaho } \\
\text { State Univ. } \\
\text { (MS\&T) }\end{array}$ & $\begin{array}{l}\text { Haiming } \\
\text { Wen }\end{array}$ & $\begin{array}{l}\text { Nanostructured } \\
\text { steels for rad. } \\
\text { tolerance }\end{array}$ & $\begin{array}{l}\text { Tensile, hardness, } \\
\text { and TEM specimens } \\
\text { for ECAP and HPT } \\
\text { (steels + HEA) }\end{array}$ & $\begin{array}{l}\text { ATR } \\
\text { (B6) }\end{array}$ & $\begin{array}{ll}\text { - } & \text { FDR comp. } \\
\text { - } & \text { Insert 2Q18 } \\
\text { - } & \text { Done 2Q21 }\end{array}$ \\
\hline 16-10393 & GE-Hitachi & $\begin{array}{l}\text { Ronald } \\
\text { Horn }\end{array}$ & $\begin{array}{l}\text { Direct Metal } \\
\text { Laser Melting } \\
\text { (DMLM) }\end{array}$ & $\begin{array}{l}\text { Tensile, CGR, fracture } \\
\text { toughness for } 316 \mathrm{~L} \\
\text { SS \& Alloy } 718(\mathrm{PH})\end{array}$ & $\begin{array}{l}\text { ATR } \\
\text { (B11) }\end{array}$ & $\begin{array}{ll}\text { - } & \text { FDR comp. } \\
\text { - } & \text { Inserted } \\
& 4 Q 17 \\
\text { - } & \text { Done 3Q18 }\end{array}$ \\
\hline $16-10584$ & $\begin{array}{l}\text { Colorado } \\
\text { School of } \\
\text { Mines }\end{array}$ & $\begin{array}{l}\text { Jeffrey } \\
\text { King }\end{array}$ & $\begin{array}{l}\text { Commercial SS } \\
\text { and Inconel }\end{array}$ & $\begin{array}{l}\text { Tensile, MPC for } 316 \mathrm{~L} \\
\text { SS \& Alloy } 718(\mathrm{PH})\end{array}$ & $\begin{array}{l}\text { ATR } \\
\text { (B5) }\end{array}$ & $\begin{array}{ll}\text { - } & \text { FDR comp. } \\
\text { - } & \text { Insert 2Q18 } \\
\text { - } & \text { Done 3Q18 }\end{array}$ \\
\hline
\end{tabular}




\section{Advanced Sensors}

\begin{tabular}{|c|c|c|c|c|c|c|}
\hline Number & Institution & PI & Purpose & Materials & $\mathbf{R x}$ & Status \\
\hline $\begin{array}{l}\text { 15-8389 } \\
\text { ULTRA-2 }\end{array}$ & $\begin{array}{l}\text { Idaho } \\
\text { National } \\
\text { Laboratory }\end{array}$ & $\begin{array}{l}\text { Joshua } \\
\text { Daw }\end{array}$ & $\begin{array}{l}\text { Sensor qualification: } \\
\text { fission gas release, } \\
\text { fission gas } \\
\text { composition, and } \\
\text { axial temp. meas. }\end{array}$ & $\begin{array}{l}\text { INL- CEA } \\
\text { pressure sensor, } \\
\text { ultrasonic } \\
\text { thermometer } \\
\text { and fiber optics }\end{array}$ & MITR-II & $\begin{array}{l}\text { Running } \\
\text { through } \\
\text { FY18 }\end{array}$ \\
\hline $17-13073$ & $\begin{array}{l}\text { Univ. of } \\
\text { Pittsburgh }\end{array}$ & $\begin{array}{l}\text { Kevin } \\
\text { Chen }\end{array}$ & $\begin{array}{l}\text { Multi-functional fiber } \\
\text { optic sensors with } \\
\text { AM components }\end{array}$ & $\begin{array}{l}3 \text { types of silica } \\
\text { FO as well as } \\
\text { commercial } \\
\text { sapphire fiber } \\
\text { sensors }\end{array}$ & MITR-II & RTI 1Q19 \\
\hline $17-12527$ & $\begin{array}{l}\text { Boise State } \\
\text { Univ. } \\
\text { (MS\&T) }\end{array}$ & $\begin{array}{l}\text { Yanliang } \\
\text { Zhang }\end{array}$ & $\begin{array}{l}\text { AM thermal sensors } \\
\text { for in-pile thermal } \\
\text { conductivity } \\
\text { measurement }\end{array}$ & $\begin{array}{l}\text { Aerosol-jet } \\
\text { printed thermal } \\
\text { conductivity } \\
\text { sensors ( } \mathrm{Pt} \\
\text { printed on } \mathrm{CeO}_{2} \text { ) }\end{array}$ & $\begin{array}{l}\operatorname{NCSU}(18) \\
\operatorname{MITR}(19)\end{array}$ & $\begin{array}{l}\text { NCSU irrad. } \\
\text { done 4Q18 }\end{array}$ \\
\hline BR2-1 & $\begin{array}{l}\text { Idaho } \\
\text { National } \\
\text { Laboratory }\end{array}$ & $\begin{array}{l}\text { Troy } \\
\text { Unruh }\end{array}$ & $\begin{array}{l}\text { Rx performance } \\
\text { benchmarking and } \\
\text { thermal modeling }\end{array}$ & $\begin{array}{l}\text { SiC Temperature } \\
\text { Monitors }\end{array}$ & BR2 & $\begin{array}{l}\text { Completed } \\
\text { irrad. 4Q17 }\end{array}$ \\
\hline BR2-4 & $\begin{array}{l}\text { Idaho } \\
\text { National } \\
\text { Laboratory }\end{array}$ & $\begin{array}{l}\text { Joshua } \\
\text { Daw }\end{array}$ & $\begin{array}{l}\text { Irradiation } \\
\text { performance of adv. } \\
\text { temp. sensors }\end{array}$ & $\begin{array}{l}\text { ultrasonic } \\
\text { thermometer } \\
\text { and fiber optics }\end{array}$ & BR2 & In design \\
\hline
\end{tabular}




\section{Accident-Tolerant Materials}

\begin{tabular}{|c|c|c|c|c|c|c|}
\hline Number & Inst. & PI & Purpose & Materials & $\mathbf{R x}$ & Status \\
\hline $16-10468$ & ORNL & Yutai Katoh & $\begin{array}{l}\text { High-heat flux } \\
\text { irradiations of SiC } \\
\text { cladding }\end{array}$ & $\begin{array}{l}\text { CVD SiC, } \\
\text { composite SiC and } \\
\text { coated SiC tubes }\end{array}$ & $\begin{array}{l}\text { HFIR } \\
\text { (rabbit) }\end{array}$ & $\begin{array}{ll}\text { - } & \text { RTI 4Q } 17 \\
\text { - } & \text { Irradiation } \\
& 2 \text { Q18 }\end{array}$ \\
\hline $16-10784$ & ORNL & $\begin{array}{l}\text { Tyler } \\
\text { Gerczak }\end{array}$ & $\begin{array}{l}\text { Radiation- } \\
\text { Enhanced } \\
\text { Diffusion of Ag, } \\
\text { Ag-Pd, Eu, and Sr } \\
\text { (TRISO) }\end{array}$ & $\begin{array}{l}\text { Diffusion couples } \\
\text { of Ion-implanted } \\
\text { PyC/SiC }\end{array}$ & $\begin{array}{l}\text { HFIR } \\
\text { (rabbit) }\end{array}$ & 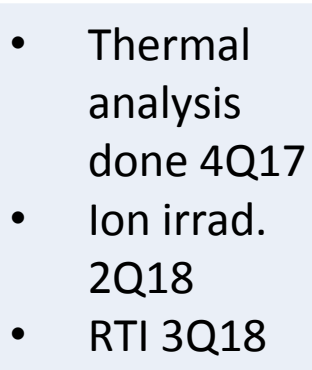 \\
\hline $17-12573$ & $\begin{array}{l}\text { General } \\
\text { Atomics }\end{array}$ & $\begin{array}{l}\text { Christian } \\
\text { Deck }\end{array}$ & $\begin{array}{l}\text { Performance of } \\
\text { SiC-SiC cladding } \\
\text { and endplug } \\
\text { Joints under } \\
\text { neutron irrad. \& } \\
\text { thermal gradient }\end{array}$ & $\begin{array}{l}\text { tube-endplug and } \\
\text { torsion joined } \\
\text { specimens of SiC- } \\
\text { SiC composites }\end{array}$ & $\begin{array}{l}\text { HFIR } \\
\text { (rabbit) }\end{array}$ & 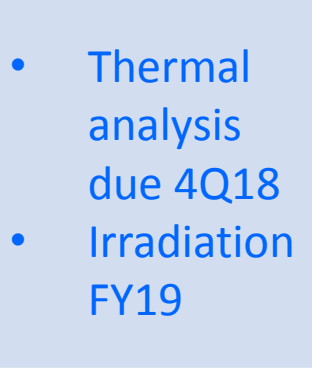 \\
\hline $17-13007$ & AREVA & $\begin{array}{l}\text { Jacqueline } \\
\text { Stevens }\end{array}$ & $\begin{array}{l}\text { ATF Neutron } \\
\text { Absorbers to } \\
\text { replace Ag-In-Cd \& } \\
\mathrm{B}_{4} \mathrm{C}\end{array}$ & $\begin{array}{l}\text { Hafnium Carbide, } \\
\text { HfC w/<3wt\% } \\
\text { MoSi2, Samarium } \\
\text { Hafnate \& } \\
\text { Europium Hafnate }\end{array}$ & $\begin{array}{l}\text { HFIR } \\
\text { (rabbit) }\end{array}$ & 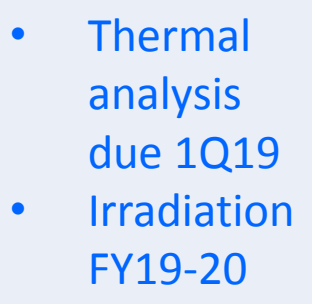 \\
\hline
\end{tabular}


Nuclear Science User Facilities

SPECIMEN READINESS ISSUES AND SOLUTIONS 


\section{Specimen Readiness Issues}

- INL-15-8389: Sensor qualification test

- delay in shipment of CEA sensors

- ISU-16-10537: Nanostructured steels for rad. tolerance

- delay in shipment of bulk material

- difficulty in fabrication of specimens from bulk material

- CSM-16-10584: Commercial AM alloys

- Difficulty in identifying material vendors 


\section{Changes to the CINR FOA}

NOTE: Applicants must demonstrate readiness for NSUF access.

- In the NSUF Access Request, a summary of readiness is required.

- In the full application, a detailed description of readiness is required.

- Awarded projects that are found to not be ready for NSUF access may be cancelled.

The following items must be completed prior to requesting access:

- Development and qualification of fabrication techniques, processes and methods

- Pre-irradiation characterization (physical, mechanical, thermal, chemical and other applicable properties)

- Material interaction studies (at irradiation conditions)

- Corrosion studies (at irradiation conditions)

NSUF/INL will arrange for fabrication of materials into specimens for the test reactor irradiations. 


\section{Changes to the CINR FOA}

A plan for delivery with specific attention to the following:

- Structural and cladding materials for neutron irradiation must be supplied to NSUF three months after project initiation..

- For previously irradiated fuels and materials not residing in the NFML, information that will be needed in order to ship and/or prepare the fuel or material for examination must be identified.

- Ownership of the materials

- For any fuels or materials supplied for the purpose of neutron irradiation, the applicant must own and have full authority to transfer ownership to DOE.

- For fuels or materials coming from other DOE programs (not NSUF), a statement of program commitment is required. 


\section{Usability Improvements}

In order to better support the users of the NSUF access programs:

- Developing web-based tools to help users and NSUF Tech Leads:

1. Irradiation resource selection

- Neutron flux and spectrum for NSUF reactors

- Most efficient allocation of resources

- Convert Neutron Fluence to DPA

- Materials scientists request dpa

- Reactor engineers think in terms of fluence

- Compound materials can be difficult

2. ATR Experiment Database

- Library of prior ATR irradiation experiment documentation

3. Estimate sample activity following irradiation

- Estimate time to be able to ship samples

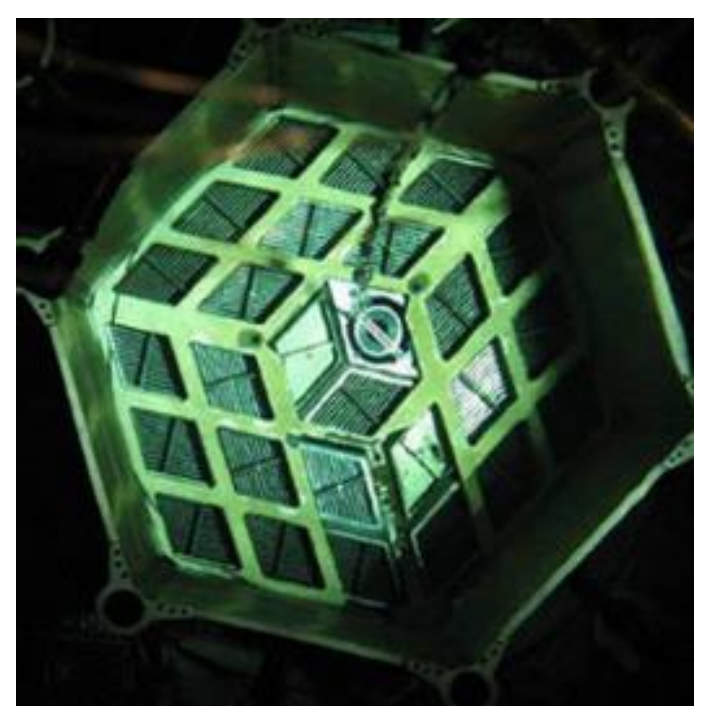

- Determine facilities that can accept materials

- Estimate dose from characterization procedures

- Also for materials in the NFML 


\section{Initiative}

In-pile Instrumentation

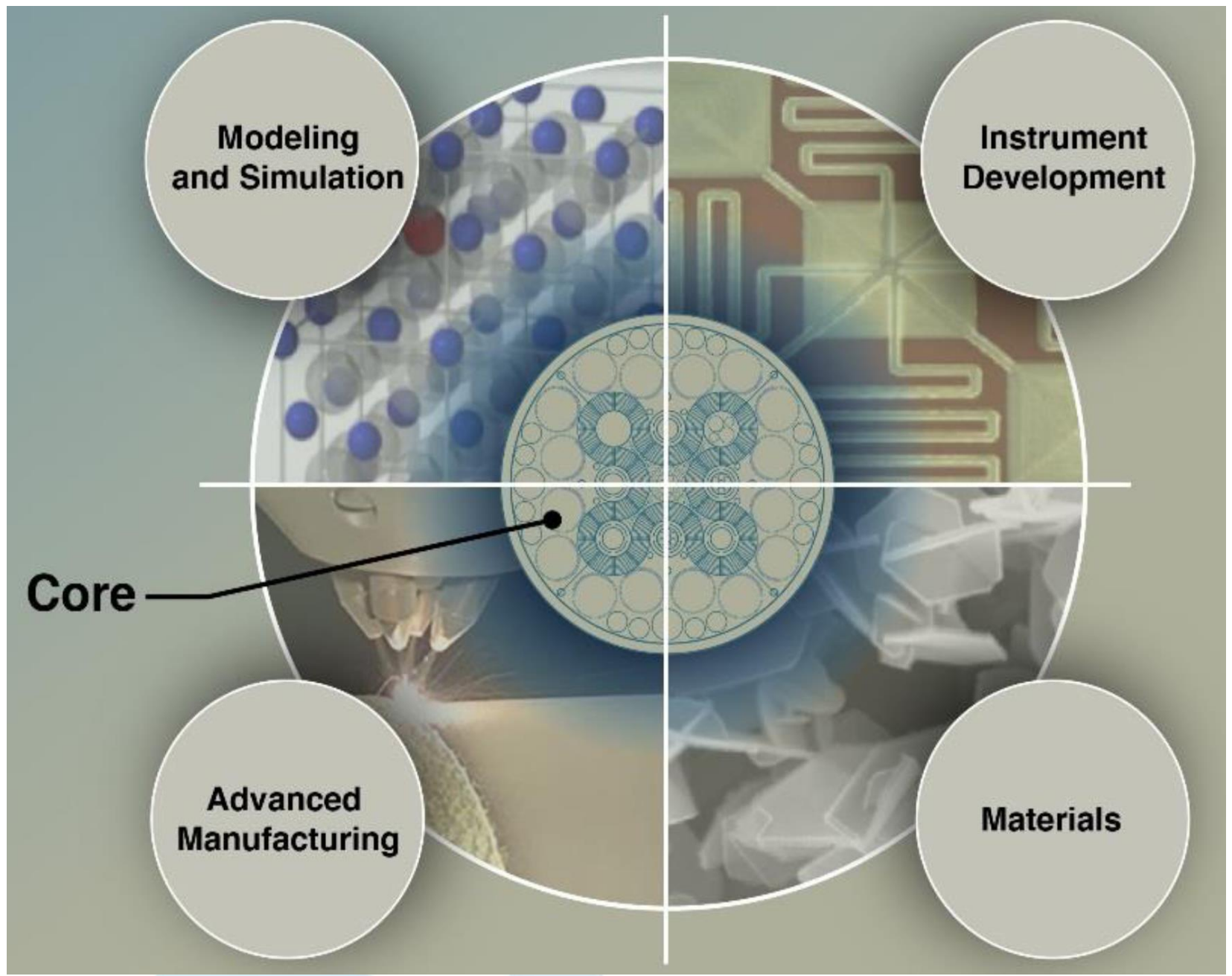




\section{Testing Strategy for Novel Materials}

Irradiation Testing Hierarchy

1. Ion Beams Irradiation Facilities

- Allow immediate feedback of performance

- Ease of instrumentation

- Ease of environmental tuning

2. Low-Power Research Reactors

- Proof-of-concept (First 1\% and 10\% testing)

- Instrumentation development (pulsing for TREAT)

- Neutron radiography

- Experiment modeling \& validation efforts

3. High-Performance Test Reactors

- Proof-of-performance

- Prototypical environment

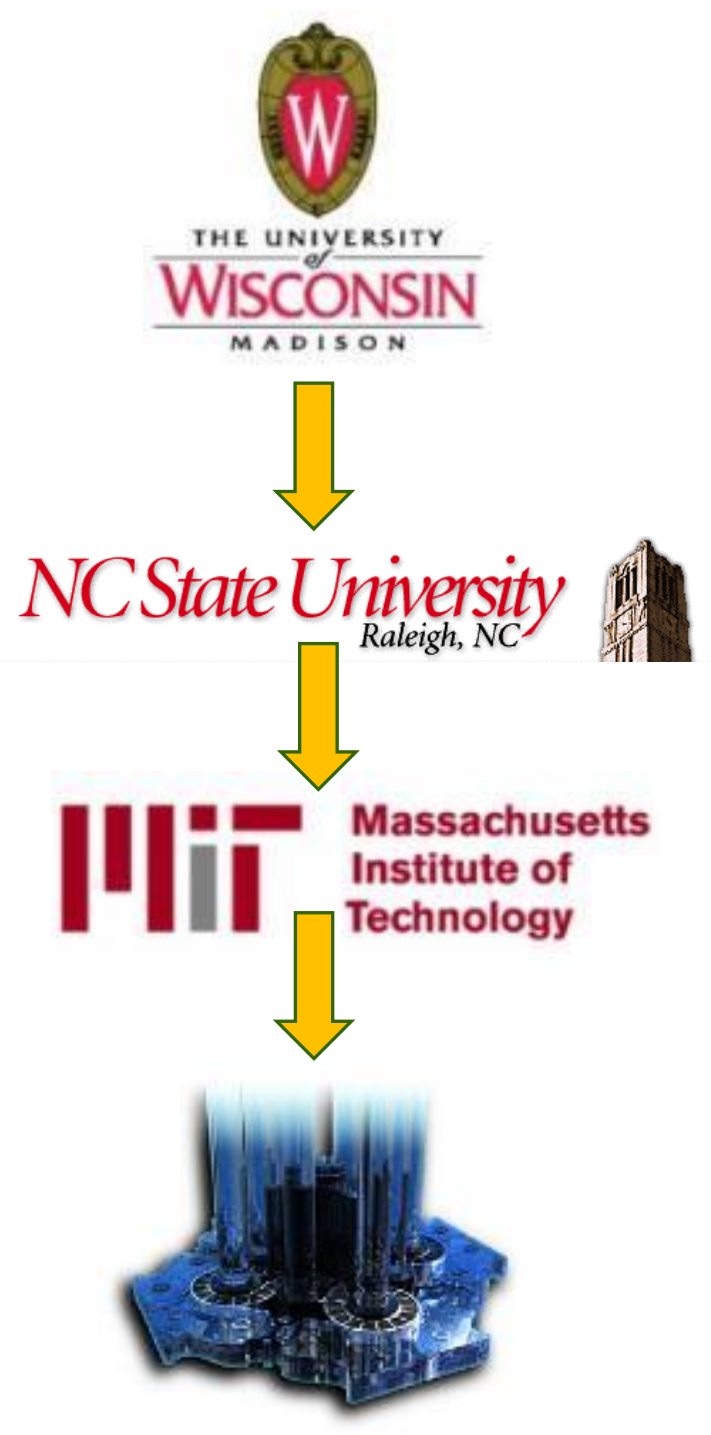




\section{Irradiation resource selection tool}

The goal of this project was a tool that NSUF users and technical project leads can use during the conceptual design phase of the proposal to select the irradiation location which is the most appropriate.

The tool has three main functions:

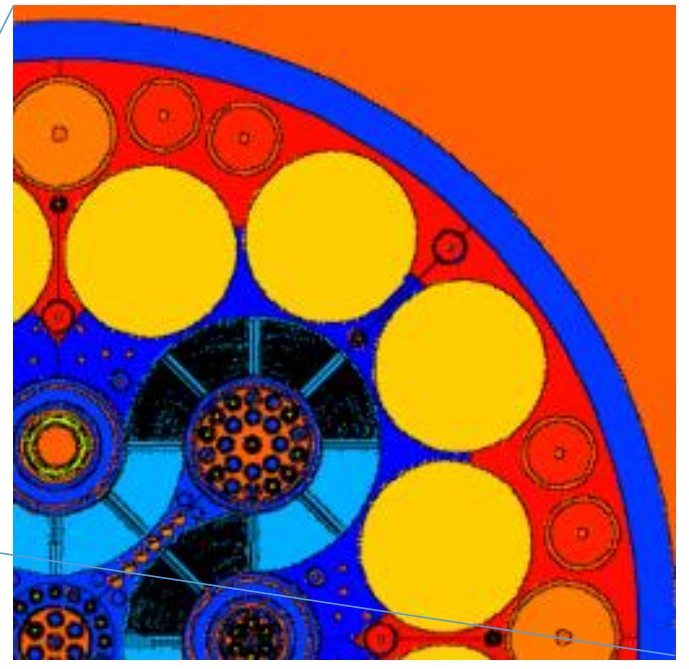

1) calculate displacements per atom (DPA) for multiple different materials,

2) calculate the time needed to reach the desired DPA, and

3) inform users what position in what reactor will give them the desired radiation damage the most effectively. 


\section{ATR Irradiation Testing Tool}

- https://nst.inl.gov/irradiationtesting

MVL boho Nebina labractory Nucleus

Nuclear Material Experiments Irradiated in the Advanced I est Reactor

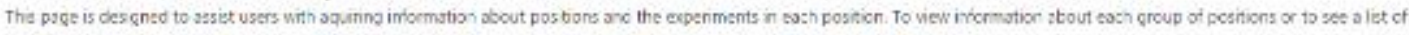

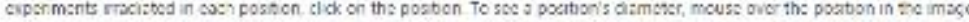

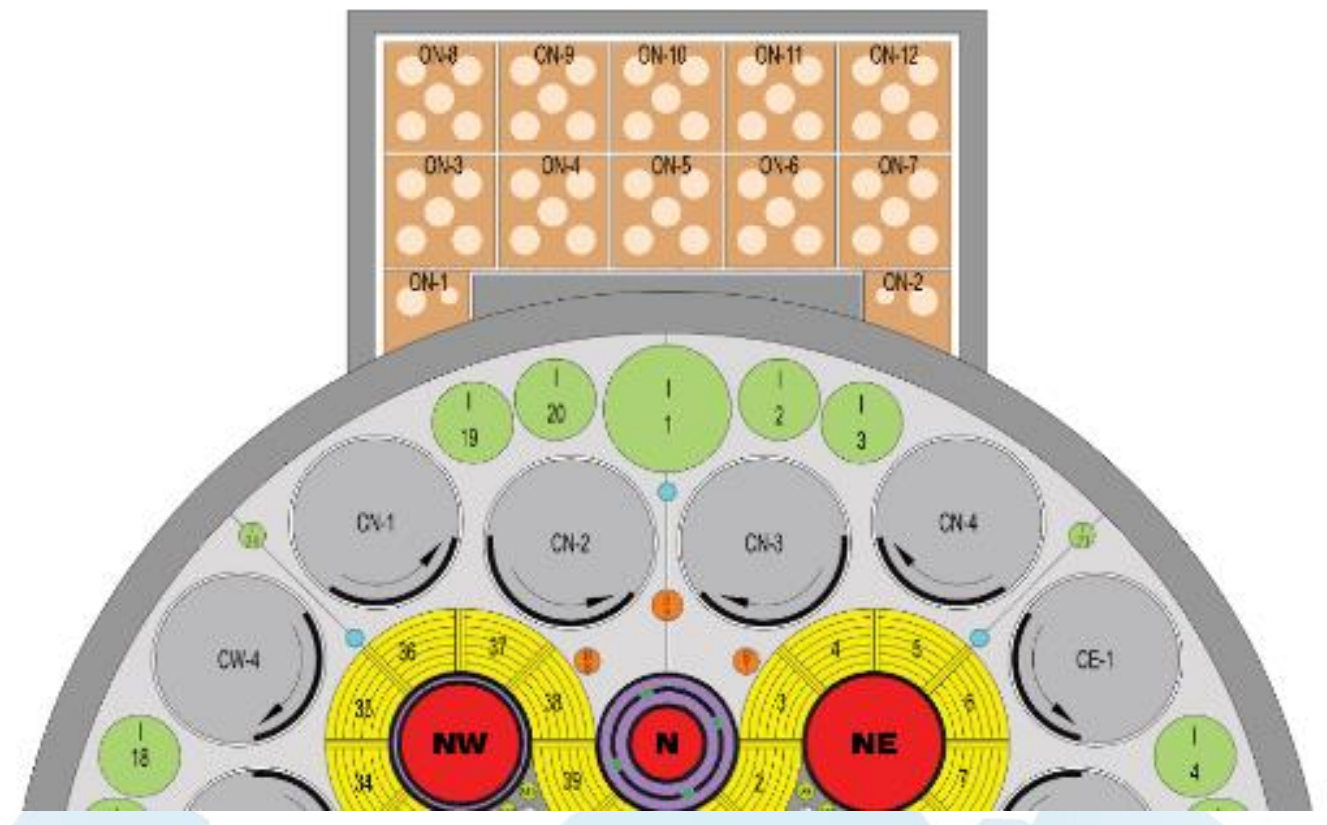

Additional Useful Tools

DPA Calculator

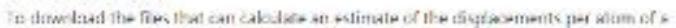

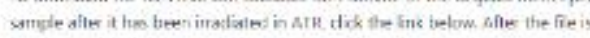

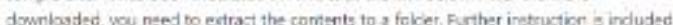
in the apsed tic under nel_man.sipdt.

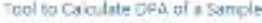

List of Experiments

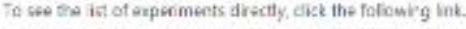

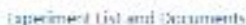

Updating Instructions

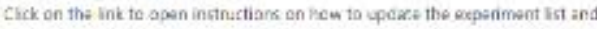
apminm-i pastions

cpdoting -uturial: Accing a New Ecperiment

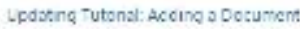

Intem Expo Presentation

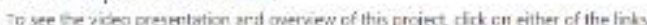
below.

Arsentestion imm:

Arsomation ime 


\section{Sample Activation Tool}

Estimating the radioactivity of a sample before it is ever irradiated will:

(1) increase awareness for worker safety,

(2) improve efficiency by planning the examination work at the appropriate facility, and reducing shipping costs

(3) inform researchers of project delays due waiting for decay.
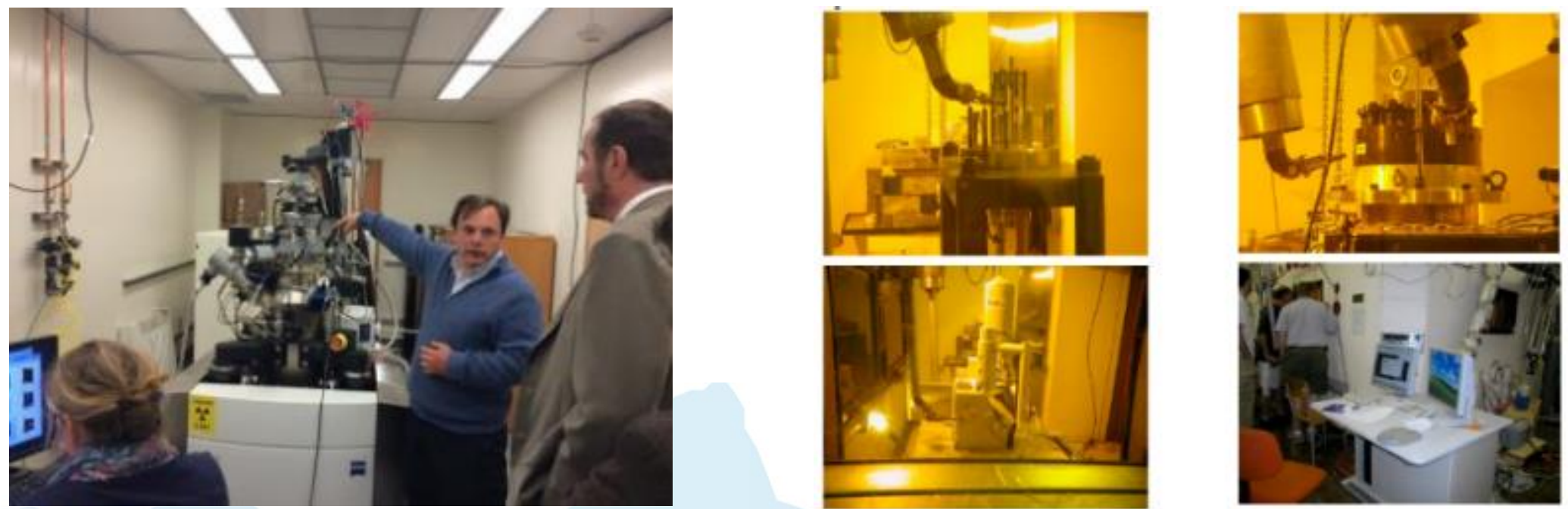


\section{Small Specimen Tensile Testing Challenge}

- Tensile testing has long been an important method for determining the material properties of different structural steel components.

- The effect of irradiation on these steel components is of particular interest to the nuclear power industry.

- The large (E8) specimens typically used are not efficient for test reactor irradiations. They also usually require a hot cell for performing postirradiation examination.

- Research into using small-scale tensile specimens has been of great interest in the nuclear industry for quite some time.
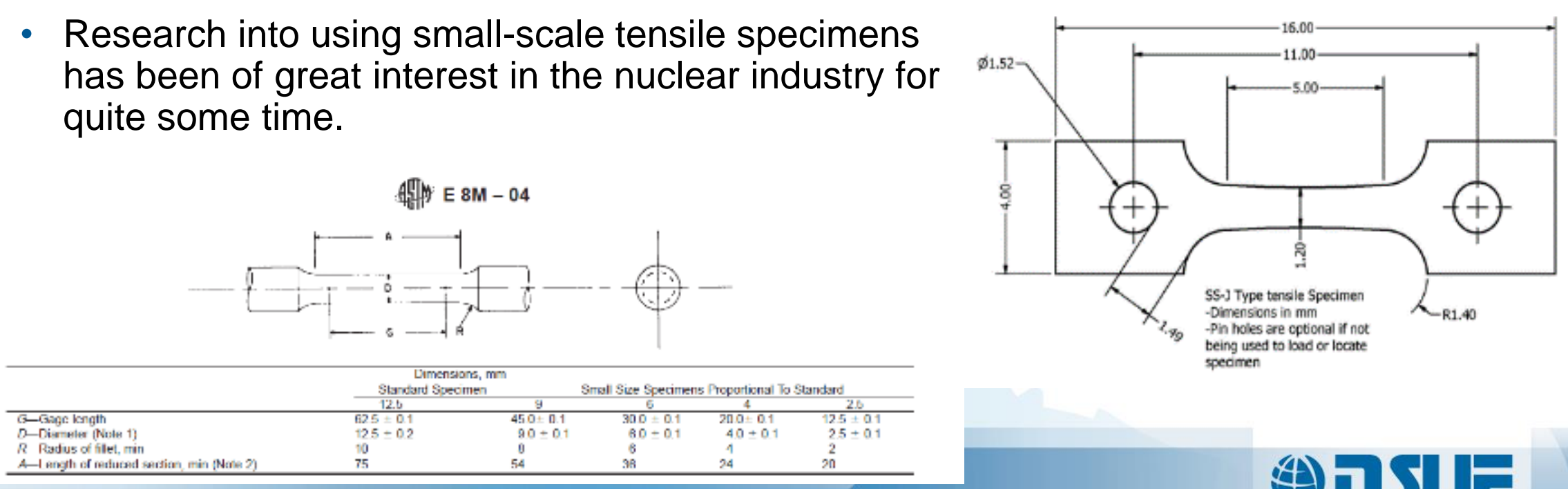

\begin{tabular}{|c|c|c|c|}
\hline \multirow{2}{*}{ Alloy } & \multicolumn{2}{|c|}{ Dose Rate } & \multirow{2}{*}{$6 \mathrm{dpa}$} \\
\cline { 2 - 3 } & $\mathrm{T}=0$ & $\mathrm{~T}=365$ & \\
\hline SA 508 & 112 & 97 & \multirow{1}{*}{} \\
\hline 625 & 75 & 28 & \\
\hline 718 & 6.2 & 0.13 & \multirow{2}{*}{$\mathrm{k} / \mathrm{hr} @ 30 \mathrm{~cm}$} \\
\hline 690 & 1.5 & 0.10 & \\
\hline $316 \mathrm{~L}$ & 3.8 & 0.10 & \\
\hline Grade 91 & 2.3 & 0.09 & \\
\hline $304 \mathrm{~L}$ & 3.8 & 0.09 & \\
\hline
\end{tabular}




\section{Future Work}

1. Couple the Irradiation Selection Tool with the Activation Tool

- IST calculates irradiation time from spectrum and power and feeds fluence and time data to AT.

- AcT uses the 100-energy group flux to calculate activation of specimen

2. Integrate these tools into the NSUF Storefront.

- Reactor selection is limited by "reasonable" irradiation times, nothing too big or too small.

- Shipping and PIE facility choices are informed by specimen radiation levels.

3. Verify results with MCNP-ORIGEN calculations 


\section{Special Session at Summer 2018 ANS Meeting, Philadelphia, PA}

"Applications of DOE-NE Infrastructure Support for University Research Reactors"

- University research reactors have formed a cornerstone of nuclear engineering research and education since the first reactor was deployed in 1954 at the NCSU.

- The population grew to a high of $~ 80$ in 1970, but has dropped to 24 in 2017.

- DOE-NE has supported the remaining reactors through fuel and infrastructure support.

- Since 2009, DOE-NE has awarded 208 proposals totaling over \$56 million for research reactor infrastructure not including fuel support.

- This session is intended to highlight the unique and innovative applications that have been funded through the DOE-NE RRI program that have helped to keep these reactors viable into the $21^{\text {st }}$ century.

- IRD wants this to be a recurring session, alternating RRU \& GSI 


\section{Contact Information for NSUF}

Brenden Heidrich

(208) 526-8117

Brenden.Heidrich@INL.gov
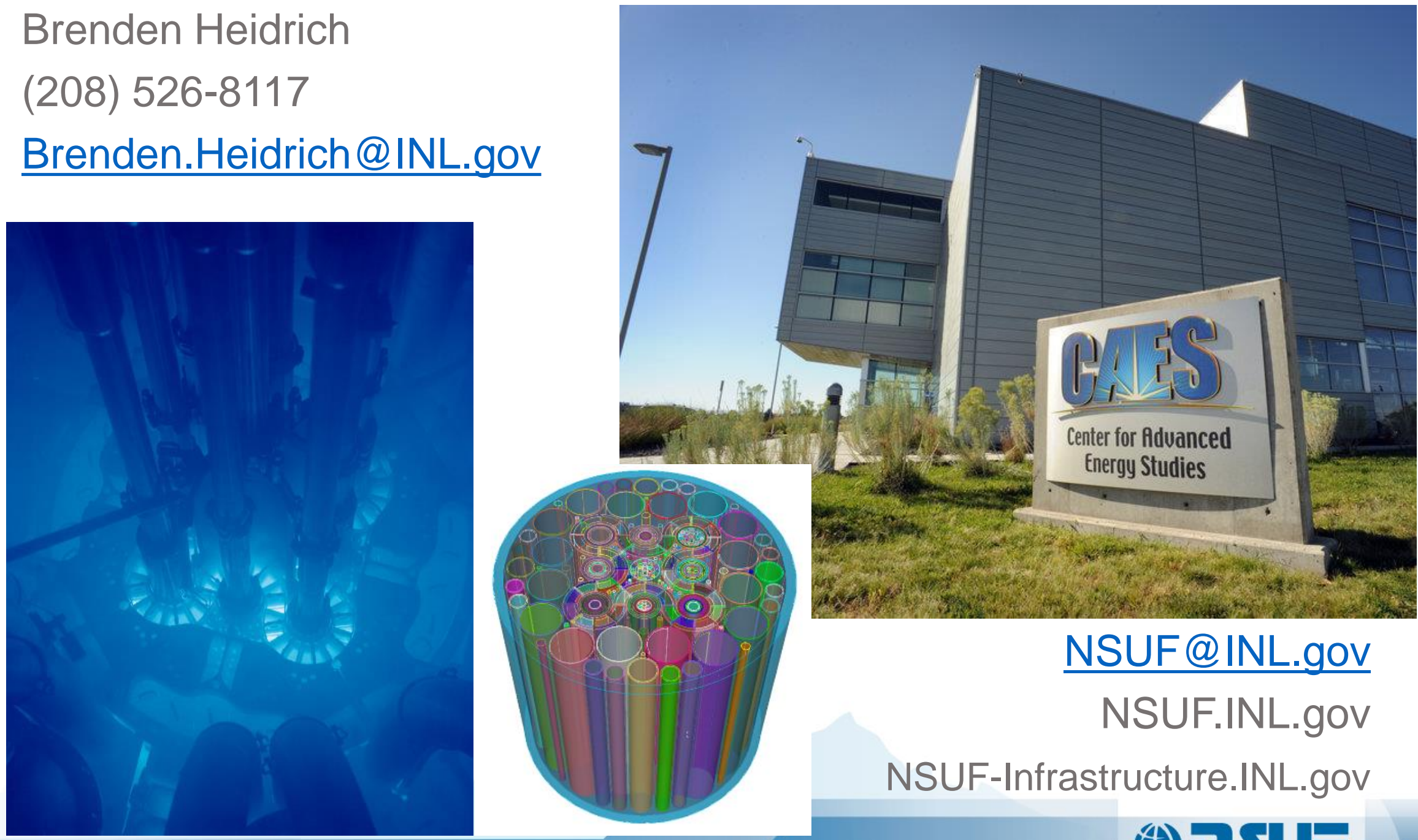

NSUF-Infrastructure.INL.gov 


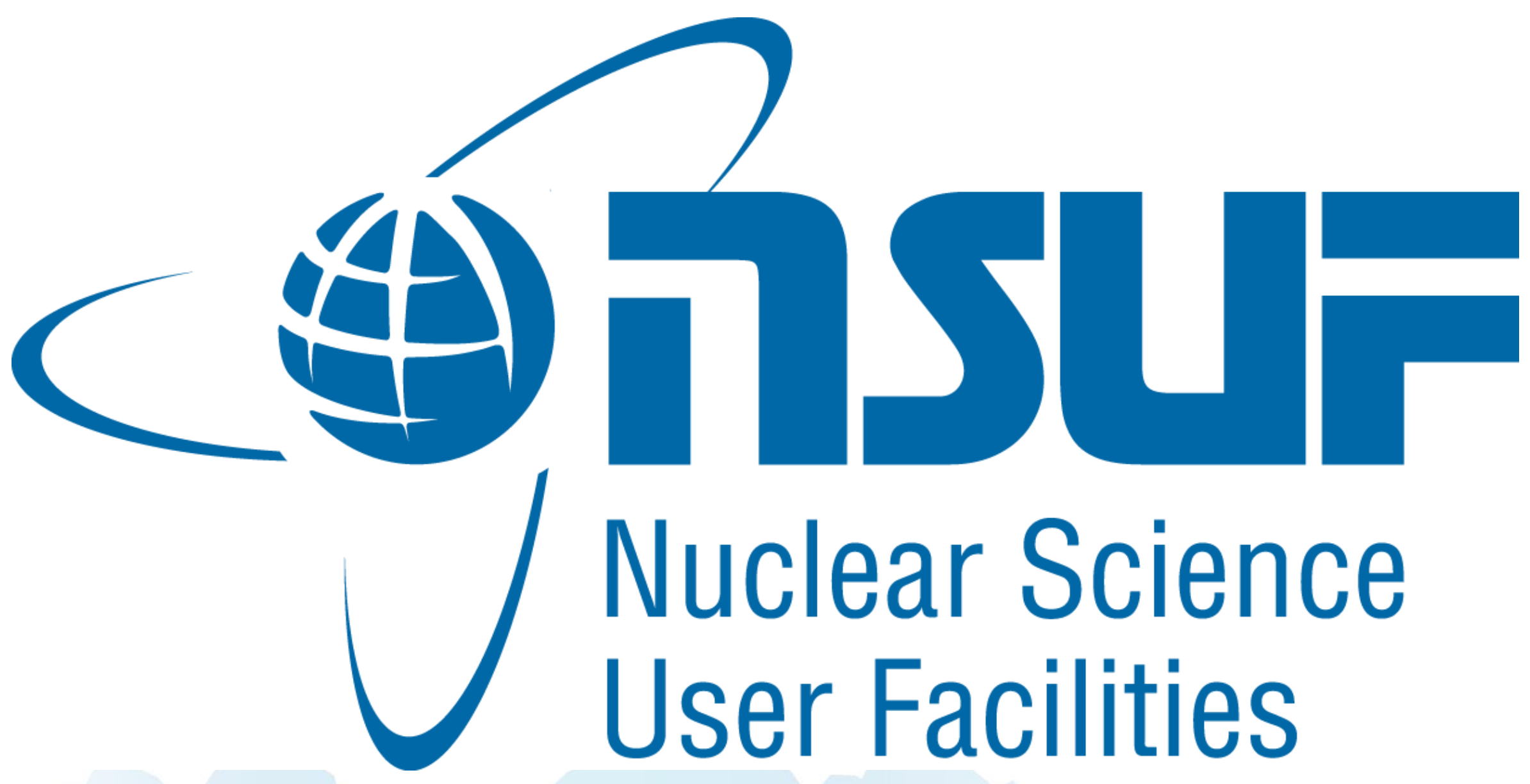
(4) 


\section{DISCLAIMER}

- This information was prepared as an account of work sponsored by an agency of the U.S. Government.

- Neither the U.S. Government nor any agency thereof, nor any of their employees, makes any warranty, express or implied, or assumes any legal liability or responsibility for the accuracy, completeness, or usefulness of any information, apparatus, product, or process disclosed, or represents that its use would not infringe privately owned rights.

- References herein to any specific commercial product, process, or service by trade name, trademark, manufacturer, or otherwise, does not necessarily constitute or imply its endorsement, recommendation, or favoring by the U.S. Government or any agency thereof.

- The views and opinions of authors expressed herein do not necessarily state or reflect those of the U.S. Government or any agency thereof. 